
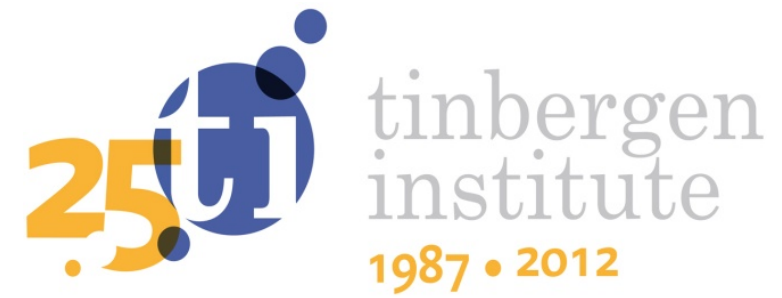

\title{
Competition in Multi-Modal Transport Networks: \\ A Dynamic Approach
}

Adriaan Hendrik van der Weijde Erik T. Verhoef

Vincent A.C. van den Berg

Faculty of Economics and Business Administration, VU University Amsterdam, and Tinbergen Institute. 
Tinbergen Institute is the graduate school and research institute in economics of Erasmus University Rotterdam, the University of Amsterdam and VU University Amsterdam.

More TI discussion papers can be downloaded at http://www.tinbergen.nl

Tinbergen Institute has two locations:

Tinbergen Institute Amsterdam

Gustav Mahlerplein 117

1082 MS Amsterdam

The Netherlands

Tel.: +31(0)205251600

Tinbergen Institute Rotterdam

Burg. Oudlaan 50

3062 PA Rotterdam

The Netherlands

Tel.: +31(0)10 4088900

Fax: $+31(0) 104089031$

Duisenberg school of finance is a collaboration of the Dutch financial sector and universities, with the ambition to support innovative research and offer top quality academic education in core areas of finance.

DSF research papers can be downloaded at: http://www.dsf.nl/

Duisenberg school of finance

Gustav Mahlerplein 117

1082 MS Amsterdam

The Netherlands

Tel.: +31(0)20 5258579 


\title{
Competition in Multi-Modal Transport Networks: A Dynamic Approach
}

\author{
Adriaan Hendrik VAN DER WEIJDE ${ }^{1}$, Erik T. VERHOEF, Vincent A. C. VAN DEN BERG \\ Department of Spatial Economics, Vrije Universiteit Amsterdam, De Boelelaan 1105, 1081HV Amsterdam, The Netherlands. \\ Tinbergen Institute, Gustav Mahlerplein 117, 1082MS Amsterdam, The Netherlands
}

31 October 2012

\begin{abstract}
We analyse the behaviour of market participants in a multi-modal commuter network where roads are not priced, but public transport has a usage fee, which is set while taking the effects on the roads into account. In particular, we analyse the difference between markets with a monopolistic public transport operator, which operates all public transport links, and markets in which separate operators own each public transport link.

To do so, we consider a simple transport network consisting of two serial segments and two parallel congestible modes of transport. We obtain a reduced form of the public transport operator's optimal fare setting problem and show that, even if the total travel demand is inelastic, serial Bertrand-Nash competition on the public transport links leads to different fares than a serial monopoly; a result not observed in a static model. This results from the fact that trip timing decisions, and therefore the generalized prices of all commuters, are influenced by all fares in the network.

We then use numerical simulations to show that, contrary to the results obtained in classic studies on vertical competition, monopolistic fares are not always higher than duopolistic fares; the opposite can also occur. We also explore how different parameters influence the price differential, and how this affects welfare.
\end{abstract}

Keywords: Public transport, congestion, market design.

JEL Classification: L10, L92, R41, R48

\section{Introduction}

A substantial literature exists on road pricing, as well as on the effects of road pricing on public transport markets, and second-best pricing of roads in the presence of unpriced substitutes. In reality, road pricing is politically difficult to implement and, with a few exceptions, roads remain unpriced. It is therefore interesting to consider the opposite, but common situation, in which roads are unpriced, but public transport has a usage fee, which is set while taking the effects on the roads into account.

Recent decades have seen a shift from governmental provision of public transport to provision by private firms, thus reducing the government's control over fares. However, not all systems have been privatized in the same way and consequently, various market structures have emerged. Although, in many countries, there is now at least some form of vertical separation between service operators and infrastructure managers, the amount of competition between service operators differs greatly. In some

\footnotetext{
${ }^{1}$ Corresponding author. Tel.: +31 (0) 205986016.

E-mail addresses: h.vander.weijde@vu.nl (A.H. van der Weijde), e.t.verhoef@vu.nl (E.T. Verhoef), v.a.c.vanden.berg@vu.nl (V.A.C. van den Berg).
} 
countries, such as The Netherlands, one operator owns the exclusive rights to operate most or even all connections in the network; in others, such as the UK, new operators can freely enter the market to offer new services, or directly compete for franchises to operate existing ones.

We therefore test how these different market structures influence public transport fares and social welfare, and how they compare with governmental provision. In particular, we analyse the difference between markets with a monopolistic public transport operator, which operates all public transport links, and markets in which separate operators own each public transport link. Standard economic theory would predict that in normal markets, both Bertrand (price) and Cournot (quantity) competition lead to lower prices than a monopoly; in the former, prices would be driven down to marginal costs when there are at least two competitors; in the latter, prices approach marginal costs only when the number of competitors approaches infinity.

Transport systems usually consist of several interacting markets, and there are unpriced externalities associated with travel, so these standard results do not always apply. Previous studies (Economides \& Salop 1992, see De Borger et al., 2008 for a transport application) have shown that, generally, parallel (or horizontal) competition, where a number of competitors offer different possibilities to travel between two points, is beneficial, both to consumers and to society. In contrast, serial (or vertical) competition, in which different operators own complementary links, increases fares and adversely affect social welfare. In this situation, each serial competitor exerts local market power, and is able to set a price above marginal cost. If the overall demand is price-sensitive, this has a negative effect on the patronage of other links, but this externality is disregarded by the individual operators; a phenomenon that is comparable to the mechanism of double marginalization.

However, these results have been obtained with static models. In these models, commuters only choose a mode, or combination of modes, to travel; the models disregard the fact that commuters can also choose the moment at which they travel. In most real-world applications, commuters do have this choice, and empirical evidence suggests that this has non-negligible effects (Small, 1982). We will therefore use dynamic modelling techniques, and examine how this affects competition. In order to do so, we assume that demand is fixed, such that we can isolate dynamic interaction effects from possible effects of pricesensitive demand.

To further improve tractability, we only examine networks in which commuters from different origins, located along one transportation corridor, travel to one destination; this may, for example, represent a morning commute from a series of suburbs to a central business district. More general network models exist (e.g. Pels \& Verhoef, 2007), but are often too complicated to yield the economic insights we are interested in. Simpler multi-modal network models (e.g. Arnott \& Yan, 2000; Verhoef, 2008) often assume that there are only two nodes in the network, that only one mode is chosen for the entire journey, or that all parallel links are exactly the same, which is too simple for our purposes. There is also some earlier literature on the properties of congested many-to-one commuter networks similar to ours (e.g. Tian et al., 2007; Arnott \& DePalma, 2010), but these are usually concerned with the user equilibrium only, and do not include competing parallel modes.

We therefore combine elements from different contributions to this literature, to model a simple dynamic multi-modal many-to-one commuter network where transfers between modes are possible, but costless. In this way, we can capture the essence of serial competition, parallel competition, mode choice and departure time choice in one analytical framework, and study the efficiency of different types of market organization. Using this model, we obtain a reduced form of the public transport operator's optimal fare setting problem, assuming that its fare is constant over time. This reduced form is 
considerably simpler and easier to use than the original optimal control problem, and we can use it to show that in this dynamic model, even though the total travel demand is inelastic, serial Bertrand-Nash competition on the public transport links leads to different fares than a serial monopoly. However, contrary to the results obtained in classic studies on vertical competition, the difference between duopolistic and monopolistic fares is not necessarily positive. We also show why these results cannot be observed in static models, and further examine these results in a series of numerical simulations.

The following section will outline the methodology and assumptions. In section 3, we examine the fare-setting behaviour of public transport operators in a static model with fixed demand, and briefly discuss why the monopolistic and serial Nash-Bertrand equilibrium fares are equal in that setting; this gives us a benchmark against which to compare the results of a dynamic model. In section 4, we then formulate this dynamic model, derive a reduced form of the public transport operator's profit optimization problem and again compare monopolistic and serial Nash-Bertrand fares. Section 5 illustrates these issues with a numerical version of the dynamic model, and examines which parameters influence the difference between the monopolistic and duopolistic fares, and thus social welfare. Section 6 concludes.

\section{Methodology}

Even our simple models require a significant amount of notation. Table 1 summarizes the main indexes, variables and parameter that will be used in our exposition.

Table 1 - Notation

\begin{tabular}{ll}
\hline \hline Indexes & \\
\hline$l$ & Segments $(1,2)$ \\
$m$ & Modes ( $R=$ road, $T=$ train) \\
$t$ & Time \\
\hline Variables \\
\hline$c_{\text {mon }}$ & Operation and maintenance costs of a monopolistic operator \\
$c_{l}$ & Operation and maintenance costs of a duopolistic operator on segment $l$ \\
$f_{l}^{m}$ & Usage fee for mode $m$ on segment $l$ (road price or rail fare) \\
$n_{l}^{m}$ & Number of commuters travelling on mode $m$, segment $l$ \\
$t_{1}^{m}$ & Arrival time at the destination of the first commuter from the first node who has used mode $m$ \\
$t_{2}^{m}$ & Arrival time at the destination of the first commuter from the second node who has used mode $m$ \\
$v_{l}^{m}$ & Arrival flow on link $l$, mode $m$ \\
$r_{l}^{m}$ & Congestion costs faced by users of mode $m$ on segment $l$ \\
$s_{l}^{m}$ & Travel speed on link $l$, mode $m$ \\
$\pi_{m o n}$ & Profit of a monopolistic operator \\
$\pi_{l}$ & Profit of a duopolistic operator on segment $l$ \\
$\theta$ & Schedule delay costs of a commuter arriving at the destination. \\
\hline Parameters \\
\hline$N$ & Number of commuters travelling from each node to the destination \\
$m$ & Segment length \\
$t^{*}$ & Desired arrival time (common to all travellers) \\
\hline \hline
\end{tabular}

To examine the effects of different market structures in a multi-modal network, we will first consider the simplest possible network in which this is possible, and later consider how the results obtained can be generalized. This simple network, shown in Fig. 1, consists of three nodes (two origins and one destination), which are connected by two segments. Each segment consists of two links. We will call these two links "rail" and "road", but they could also represent other modes, as long as they are completely separate, such that commuters using one node do not influence the travel costs of the 
commuters on the other node. Importantly, we ignore the discrete nature of public transport, and instead assume that passengers can depart and arrive continuously. Although it is theoretically possible to include a limited number of departures, this major complication would not qualitatively affect the results. ${ }^{2}$

Travellers treat the two modes as perfect substitutes, so that Wardropian equilibrium conditions apply for used alternatives; their generalized prices should be equal in equilibrium, and there are no unused alternatives with a lower price (Wardrop, 1952). If modes were imperfect substitutes, this would reduce the effects of competition, but it would not eliminate them.

We examine a typical morning commute, in which $N$ commuters travel from node 1 to the destination, and another $N$ commuters from node 2 to the destination. ${ }^{3}$ Commuters from node 1 can transfer to a different mode at node 2, although, as we will see in sections 3 and 4 below, this assumption does not influence the results in an interior equilibrium; it is also possible to disallow all transfers or only allow transfers in one direction. Transfers are costless since, in an interior equilibrium, a positive transfer cost would simply eliminate all transfers. The total number of commuters traveling from each node, $N$, is fixed, as we want to exclude the effects of elastic demand from our analysis, and in particular from complicating the comparison of equilibrium use levels across alternative market configurations.

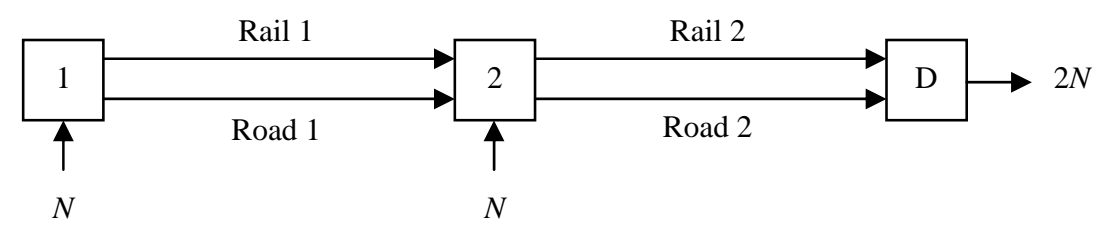

Figure 1 - Network

As a benchmark, we first consider a static model, in which departure time decisions are therefore ignored; the congestion costs commuters face are influenced by all commuters using the same links. Afterwards, we will examine a dynamic model, in which commuters choose a departure time to minimize the total cost of travelling, and only the number commuters travelling on the same links at the same time influences the congestion cost they face. In that model, there is flow congestion on the road, as in Chu (1995), a reformulation of Henderson (1974); travel speeds of commuters depend on the density of the traffic flow at the moment of arrival, where there are no specific restrictions on the functional form of this relation. This assumption allows us to obtain reduced-form formulations that could not have been obtained if other forms of congestion, such as bottleneck congestion, would have been present.

In the general formulation, we only examine interior solutions, in which all modes are used from all origins; where necessary, we assume that such a solution exists. The numerical analysis allows for all possible equilibria, and thus for the possibility that one or more links may remain unused in equilibrium.

\section{Static model}

\subsection{Generalized prices}

Commuters travelling over segment $l$ pay a road price $f_{l}^{R}$ or rail fare $f_{l}^{T}$, where the index $T$ stands for train travel, and $R$ for road travel. In addition to that, they also face a congestion $\operatorname{cost} r_{l}^{m}, m \in\{T, R\}$,

\footnotetext{
${ }^{2}$ Especially if passengers do not know the schedule and headways are exogenous; in that case, each passenger would simply incur an additional cost, equal to the value of waiting time multiplied by the expected waiting time. If passengers know the schedule or headways are endogenous, solutions will be very difficult to obtain (See also Tian et al., 2007).

${ }^{3}$ This assumed equality of commuter numbers simplifies notation, but could otherwise easily be dropped.
} 
which is a function of the number of commuters travelling on that same link, $n_{l}^{m}$. Since there is no time dimension in the static model, schedule delay costs are not defined.

\subsection{Rail monopoly}

A monopolistic rail operator, which operates both rail segments, maximizes the sum of the revenues of both links minus operating and maintenance costs $c_{\text {mon }}$ :

$\max \pi_{\text {mon }}=f_{1}^{T} n_{1}^{T}+f_{2}^{T} n_{2}^{T}-c_{\text {mon }}\left(n_{1}^{T}, n_{2}^{T}\right)$

There are two Wardropian user equilibrium constraints for commuters from the upstream node, as they can follow three possible mode choice patterns (train-train, car-car and car-train), and one constraint ensuring user equilibrium for commuters from the downstream node, as they can only choose between two modes (train or car). Two other constraints apply, ensuring that all commuters from each origin travel. The Lagrangian for profit optimization can therefore be written as

$$
\begin{aligned}
L_{\text {mon }}=f_{1}^{T} n_{1}^{T}+ & f_{2}^{T} n_{2}^{T}-c_{m o n}\left(n_{1}^{T}, n_{2}^{T}\right)+\lambda_{1}\left[r_{1}^{T}\left(n_{1}^{T}\right)+f_{1}^{T}-r_{1}^{R}\left(n_{1}^{R}\right)-f_{1}^{R}\right] \\
& +\lambda_{2}\left[r_{2}^{T}\left(n_{2}^{T}\right)+f_{2}^{T}-r_{2}^{R}\left(n_{2}^{R}\right)-f_{2}^{R}\right]+\lambda_{3}\left[n_{1}^{T}+n_{1}^{R}-N\right]+\lambda_{4}\left[n_{2}^{T}+n_{2}^{R}-2 N\right]
\end{aligned}
$$

where $\lambda_{1}$ to $\lambda_{4}$ are the Lagrange multipliers associated with the constraints mentioned above. Combining and simplifying the first-order conditions yields the optimal monopolistic rail prices:

$f_{l}^{T}=\frac{\partial c_{m o n}}{\partial n_{l}^{T}}+n_{l}^{T} \frac{\partial r_{l}^{T}}{\partial n_{l}^{T}}+n_{l}^{T} \frac{\partial r_{l}^{R}}{\partial n_{l}^{R}}$

Hence, the price on each link consists of the marginal cost of accommodating an extra commuter on that link, and a congestion-related mark-up. This mark-up consists of two parts. The second term in Eq. (3) is the increase in congestion costs that all rail users experience as a result of an increase in the number of users; naturally, the monopolist internalizes these costs. The third term in Eq. (3), which also internalizes part of the congestion on the road, is the equivalent of a standard monopolistic mark-up in a market with price-sensitive demand. With an inverse demand function $d_{l}^{T}\left(n_{l}^{T}\right)$ and in absence of an alternative mode, this mark-up would be equal to $-n_{l}^{T}\left(\partial d_{l}^{T} / \partial n_{l}^{T}\right)$. Because, in our model, any rail traveller shifted away from rail to road, the term $\partial r_{l}^{T} / \partial n_{l}^{T}$ in (3) is equivalent to what $-\partial d_{l}^{T} / \partial n_{l}^{T}$ would be in this conventional setting.

\subsection{Rail Bertrand-Nash duopoly}

If the two rail links are owned by separate operators, each operator maximizes its own profits, while both maximization problems are subject to the same constraints as those applying for the monopolistic operator. Hence, the two Lagrangians are:

$$
\begin{aligned}
& L_{b 1}=f_{1}^{T} n_{1}^{T}-c_{1}\left(n_{1}^{T}\right)+\lambda_{b 1}\left[r_{1}^{T}\left(n_{1}^{T}\right)+f_{1}^{T}-r_{1}^{R}\left(n_{1}^{R}\right)-f_{1}^{R}\right]+\lambda_{b 2}\left[r_{2}^{T}\left(n_{2}^{T}\right)+f_{2}^{T}-r_{2}^{R}\left(n_{2}^{R}\right) N-f_{2}^{R}\right] \\
& +\lambda_{b 3}\left[n_{1}^{T}+n_{1}^{R}-N\right]+\lambda_{b 4}\left[n_{2}^{T}+n_{2}^{R}-2 N\right] \\
& L_{b 2}=f_{2}^{T} n_{2}^{T}-c_{2}\left(n_{2}^{T}\right)+\gamma_{b 1}\left[r_{1}^{T}\left(n_{1}^{T}\right)+f_{1}^{T}-r_{1}^{R}\left(n_{1}^{R}\right)-f_{1}^{R}\right]+\gamma_{b 2}\left[r_{2}^{T}\left(n_{2}^{T}\right)+f_{2}^{T}-r_{2}^{R}\left(n_{2}^{R}\right) N-f_{2}^{R}\right] \\
& +\gamma_{b 3}\left[n_{1}^{T}+n_{1}^{R}-N\right]+\gamma_{b 4}\left[n_{2}^{T}+n_{2}^{R}-2 N\right]
\end{aligned}
$$

Where $\lambda_{b 1}-\lambda_{b 4}$ and $\gamma_{b 1}-\gamma_{b 4}$ are Lagrangian multipliers. The sum of the two duopolists' operation and maintenance costs may be larger than those of the monopolist if the latter can exploit economies of scale, so $c_{1}+c_{2} \geq c_{\text {mon }}$. The first-order conditions yield:

$f_{l}^{T}=\frac{\partial c_{l}}{\partial n_{l}^{T}}+n_{l}^{T} \frac{\partial r_{l}^{T}}{\partial n_{l}^{T}}+n_{l}^{T} \frac{\partial r_{l}^{R}}{\partial n_{l}^{R}}$

With the exception of the first term, Eq. (6) is equal to Eq. (3); contrary to a standard Bertrand duopoly, both operators can raise their process above marginal production cost $c_{l}^{T}$, for two reasons. The first is that 
they internalize the congestion externality on their own link, and collect the revenue from the associated toll. The second is that the serial setup implies that they are not offering pure substitutes, but rather complements, so that the parallel road is their direct competition; not the other duopolists.

\subsection{Price comparison}

If the monopolistic operator does not benefit from economies of scale in the number of rail links its owns, and thus $\frac{\partial c_{l}}{\partial n_{l}^{T}}=\frac{\partial c_{m o n}}{\partial n_{l}^{T}}$, Eqs. (3) and (6) are equal; there is no difference between Bertrand-Nash and monopolistic prices on . Only if $\frac{\partial c_{l}}{\partial n_{l}^{T}}>\frac{\partial c_{m o n}}{\partial n_{l}^{T}}$, which may be the case if a monopolistic operator can save costs as a result of owning both links, it is possible that monopolistic and duopolistic fares are different. Since the exact parameters of the model determine whether this happens or not, we will ignore this case in the analyses below.

To some extent, the above results are intuitive. All users have to travel, and all commuters from node 1 (the upstream node) pass through node 2, where they can take the train regardless of the mode they used to arrive there. Hence, the number of train travellers between each pair of nodes does not depend on the rail fare on the other link. In other words, a duopolistic operator does not have to take into account how its customers arrived at the start of its segment or how they will depart at the end, nor how much this costs the customers, since the number of customers arriving at and departing from each segment is fixed. The monopolistic optimization problem is therefore perfectly separable in the two segments, and the two duopolists set exactly the same prices as the monopolist.

So far, we have allowed commuters from node 1 to transfer between modes in either way, by not placing any restrictions on $n_{2}^{T}$ and $n_{2}^{R}$. However, transfers between modes do not have to be considered explicitly; it can be shown mathematically that the inclusion of an additional constraint specifying that $n_{2}^{T}>n_{1}^{T}$ (such that only transfers from road to train are possible) or $n_{2}^{T}>n_{1}^{T}$ and $n_{2}^{R}>n_{1}^{R}$ (such that no transfers are possible) does not change the results, as these constraints do not include the rail fares, and will apply to both duopolists and to the monopolist. The reason that the fare setting problem is still separable, even when transfers are not possible, is that commuters from the downstream node equalize the generalized price of travel on both modes on the downstream segment. Hence, when commuters from the upstream node decide which mode to use, it is sufficient for them to only consider the road prices and rail fares on the upstream segment, since the full price of travelling on the downstream segment is, thanks to the downstream commuters, independent of the chosen mode.

\section{Dynamic model}

The static model above does not allow commuters to choose a departure time that minimizes their generalized travel price. Although this may be realistic in some settings, departure time choices cannot be ignored in most real-world situations, especially in the context of congestion, and it is important to consider if the model outcomes are different if departure time choices are indeed included.

The full dynamic formulation of the problem outlined above yields a complex optimal control problem, as rail operators should now maximize the time integral of profits on each link, subject to integral constraints and temporal Wardropian equilibrium conditions. However, it is possible to derive a reduced-form formulation, under the assumption that fares are constant over time, as we will do here. In order to do this, we first establish the arrival order of commuters from the different origins and modes. We then divide the time between the arrival of the first and last commuters at the destination in four 
periods, and subsequently solve the resulting reduced-form model as if it were a static optimization problem.

\subsection{Generalized prices and arrival order}

As before, users face road prices or rail fares, and a congestion cost $r_{l}^{m}$. In the dynamic model, however, the congestion costs are a function of the number of commuters arriving at the same time, so $r_{l}^{m}(t)=r_{l}^{m}\left(v_{l}^{m}(t)\right)$. In addition, commuters also face a schedule delay cost $\theta$, which increases as commuters arrive further away from their preferred arrival time $t^{*}$. For simplicity, we assume that late arrivals (after $t^{*}$ ) are not allowed. ${ }^{4}$ We also assume that the free-flow travel speed of a car is higher than the speed of a train; this allows for establishment of the arrival order.

Define $t_{1}^{R}$ as the time the first road-road commuter from node 1 arrives at the destination, and $t_{2}^{R}$ the time the first road commuter from node 2 arrives. To ensure user equilibrium, $\frac{\partial r_{1}^{R}}{\partial v_{1}^{R}} \frac{\partial v_{1}^{R}}{\partial t}+\frac{\partial r_{2}^{R}}{\partial v_{2}^{R}} \frac{\partial v_{2}^{R}}{\partial t}+$ $\frac{\partial \theta}{\partial t}=0$ when commuters from node 1 are travelling, and $\frac{\partial r_{2}^{R}}{\partial v_{2}^{R}} \frac{\partial v_{2}^{R}}{\partial t}+\frac{\partial \theta}{\partial t}=0$ when commuters from node 2 are travelling, such that no commuters want to change their arrival time. Moreover, generalized prices faced by users from both nodes have to be lower when they are travelling then when they are not travelling. Using these facts, it can be shown that $t_{2}^{R}>t_{1}^{R}$, that the flow of road-road commuters from node 1 is positive from $t_{1}^{R}$ to $t^{*}$ and that the flow of road commuters from node 2 is positive from $t_{2}^{R}$ to $t^{*}$. Moreover, as soon as road commuters from node 2 start to arrive, the arrival flow of commuters from node 1 becomes constant. ${ }^{5}$

Define $t_{1}^{T}$ as the time the first rail-rail commuter from node 1 arrives at the destination, and $t_{2}^{T}$ the time the first rail commuter from node 2 arrives. Assuming that the free-flow travel speed on the road is higher than or equal to the travel speed of the train, $t_{1}^{T} \geq t_{1}^{R}$. As on the road, the first upstream commuter arrives first, so $t_{2}^{T}>t_{1}^{T}$. Hence, $t_{1}^{R} \leq t_{1}^{T}, t_{2}^{R}, t_{2}^{T}<t^{*}$. Fig. 2 shows an example of the user equilibrium arrival flows in model with linear congestion costs and linear costs of schedule delay.

Using these insights, we can divide period between the first and last arrival into four partially overlapping periods; two for road travel and two for rail travel, as summarized in Table 2. Within each time period, the generalized price faced by commuters using the indicated mode is constant, to satisfy the Wardropian user equilibrium constraints.

\begin{tabular}{ccc}
\multicolumn{3}{c}{ Table 2 - Time periods } \\
\hline \hline \multirow{2}{*}{ Road } & RI & $t_{1}^{R} \leq t<t_{2}^{R}$ \\
& RII & $t_{2}^{R} \leq t \leq t^{*}$ \\
\hline \multirow{2}{*}{ Train } & TI & $t_{1}^{T} \leq t<t_{2}^{T}$ \\
& TII & $t_{2}^{T} \leq t \leq t^{*}$ \\
\hline \hline
\end{tabular}

\footnotetext{
${ }^{4}$ It is also possible to allow late arrivals, which is more realistic, but this complicates the analysis without yielding additional insights. In line with some of the previous literature, (e.g. Arnott \& Kraus, 1993, 1995; Kraus \& Yoshida, 2002; Kraus (2003); van den Berg \& Verhoef (2011)) late arrivals are therefore prohibited.

${ }^{5}$ Tian et al. (2007) more rigorously show some of these properties for a single-mode many-to-one network with discrete departure times. Arnott \& DePalma (2011) do the same for a single-mode many-to-one network where origins are distributed continuously.
} 


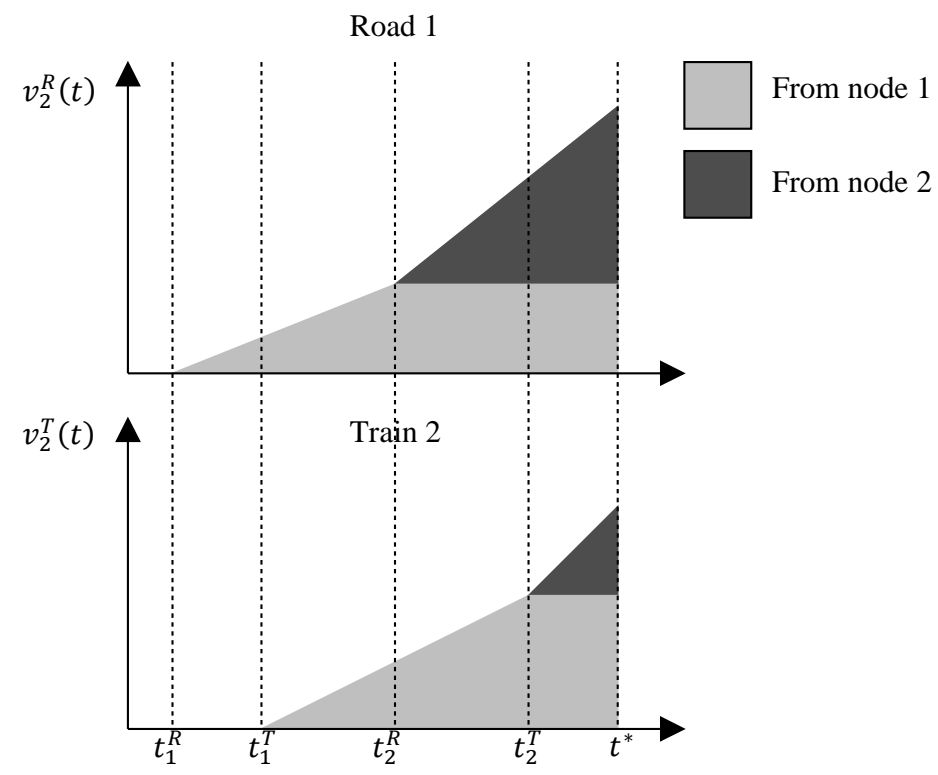

Figure 2 - Equilibrium arrival order and arrival flows at destination

\subsection{Price setting - monopolistic rail operator}

Period RI

As explained above, the generalized user price for road-road commuters from the upstream node arriving at the destination at time $t$ is given by:

$f_{1}^{R}+f_{2}^{R}+r_{1}^{R}\left(v_{1}^{R}\left(t-\frac{m}{s_{2}^{R}(t)}\right)\right)+r_{2}^{R}\left(v_{2}^{R}(t)\right)+\theta(t ; \beta)$

Note that commuters arriving at the destination at time $t$ enter the second segment at time $t-\frac{m}{s_{2}^{R}(t)}$, as $\frac{m}{s_{2}^{R}(t)}$ is the time needed to traverse the second segment; hence, congestion costs, which are assumed to be a function of the flow of commuters out of the segment, are incurred at that time.

In a Wardropian equilibrium, these generalized prices are time-invariant, so the time-derivative of (8) is equal to zero during the whole period. Moreover, since there are no commuters from another node using the road in this period and both links are identical, each individual commuter's speed is constant along the whole route, so $v_{1}^{R}\left(t-\frac{m}{s_{2}^{R}(t)}\right)=v_{2}^{R}(t)$, and therefore

$\partial v_{1}^{R}\left(t-\frac{m}{s_{2}^{R}(t)}\right) / \partial t=\partial v_{2}^{R}(t) / \partial t=-\frac{\partial \theta(t)}{\partial t} /\left[\frac{\partial r_{1}^{R}}{\partial v_{1}^{R}}+\frac{\partial r_{2}^{R}}{\partial v_{2}^{R}}\right]$

An expression for the road travel flow in this period can be obtained by integrating (9):

$v_{1}^{R}\left(t-\frac{m}{s_{2}^{R}(t)}\right)=v_{2}^{R}(t)=-\int \frac{\partial \theta(t)}{\partial t} /\left[\frac{\partial r_{1}^{R}}{\partial v_{1}^{R}}+\frac{\partial r_{2}^{R}}{\partial v_{2}^{R}}\right] d t$

where, by construction, $v_{1}^{R}\left(t_{1}^{R}-\frac{m}{s_{2}^{R}\left(t_{1}^{R}\right)}\right)=v_{2}^{R}\left(t_{1}^{R}\right)=0$; there are no arrivals before $t_{1}^{R}$. Using this, the total numbers of commuters using both roads during this interval, $N_{1}^{R I}$ (for the first segment) and $N_{2}^{R I}$ (for the second segment) can be written as the integral of the arrival flow, which is now a function of $t_{1}^{R}$ and $t_{2}^{R}$ only: 
$N_{1}^{R I}=N_{2}^{R I}=\int_{t_{1} R}^{t_{2}^{R}} v_{2}^{R}(t) d t=N_{1}^{R I}\left(t_{1}^{R}, t_{2}^{R}\right)$

\section{Period TI}

In this period, the generalized user costs for train-train commuters are constant over time. Moreover, since only commuters from the upstream node use the train, $v_{1}^{T}\left(t-\frac{m}{s_{2}^{T}(t)}\right)=v_{2}^{T}(t) . v_{1}^{T}$ and $v_{2}^{T}$ are therefore given by:

$v_{1}^{T}\left(t-\frac{m}{s_{2}^{T}(t)}\right)=v_{2}^{T}(t)=-\int \frac{\partial \theta(t)}{\partial t} /\left[\frac{\partial r_{1}^{T}}{\partial v_{1}^{T}}+\frac{\partial r_{2}^{T}}{\partial v_{2}^{T}}\right] d t$

where, by construction, $v_{1}^{T}\left(t_{1}^{T}-\frac{m}{s_{2}^{T}\left(t_{1}^{T}\right)}\right)=v_{2}^{T}\left(t_{1}^{T}\right)=0$

Hence, the total number of commuters travelling by train on the first and second segment, respectively, can be written as:

$N_{1}^{T I}=N_{2}^{T I}=\int_{t_{1}^{T}}^{t_{2}^{T}} v_{2}^{T}(t) d t=N_{1}^{T I}\left(t_{1}^{T}, t_{2}^{T}\right)$

\section{Period RII}

Now, the generalized user costs for road-road commuters from the upstream node and road commuters from the downstream node are constant over time, and equal to those of users in the previous period. Moreover, the flow of road-road commuters from the upstream node is constant, so $\partial v_{1}^{R}\left(t-\frac{m}{s_{2}^{R}(t)}\right) / \partial t=0$.

Hence, the total number of commuters using the first road segment in this period can be obtained through multiplication of the commuter flow at $t_{2}^{R}-\frac{m}{s_{2}^{R}\left(t_{2}^{R}\right)}$ (since commuters exiting the first segment at that time will arrive at their destination at $t_{2}^{R}$ ) with the length of the period:

$$
N_{1}^{R I I}=\left(\left(t^{*}-\frac{m}{s_{2}^{R}\left(t^{*}\right)}\right)-\left(t_{2}^{R}-\frac{m}{s_{2}^{R}\left(t_{2}^{R}\right)}\right)\right) v_{1}^{R}\left(t_{2}^{R}-\frac{m}{s_{2}^{R}\left(t_{2}^{R}\right)}\right)=N_{1}^{R I I}\left(t_{1}^{R}, t_{2}^{R}\right)
$$

On the downstream link, $\frac{\partial v_{2}^{R}(t)}{\partial t}=-\frac{\partial \theta(t)}{\partial t} / \frac{\partial r_{2}^{R}}{\partial v_{2}^{R}}$, and therefore

$v_{2}^{R}(t)=-\int \frac{\partial \theta(t)}{\partial t} / \frac{\partial r_{2}^{R}}{\partial v_{2}^{R}} d t$

where $v_{2}^{R}\left(t_{2}^{R}\right)$ is given by Eq. 10 .

and thus,

$N_{2}^{R I I}=\int_{t_{2}^{R}}^{t^{*}} v_{2}^{R}(t) d t=N_{2}^{R I I}\left(t_{1}^{R}, t_{2}^{R}\right)$

\section{Period TII}

Here, the generalized user costs for all types of commuters are constant over time, and equal to those of users in the previous period. Moreover, $\partial v_{1}^{T}\left(t-\frac{m}{s_{2}^{T}(t)}\right) / \partial t=0$. Hence,

$N_{1}^{T I I}=\left[\left[t^{*}-\frac{m}{s_{2}^{T}\left(t^{*}\right)}\right]-\left[t_{2}^{T}-\frac{m}{s_{2}^{T}\left(t_{2}^{T}\right)}\right]\right] v_{1}^{T}\left(t_{2}^{T}-\frac{m}{s_{2}^{T}\left(t_{2}^{T}\right)}\right)=N_{1}^{T I I}\left(t_{1}^{T}, t_{2}^{T}\right)$ 
$N_{2}^{T I I}=\int_{t_{2}^{T}}^{t^{*}} v_{2}^{T}(t) d t=N_{2}^{T I I}\left(t_{1}^{T}, t_{2}^{T}\right)$

Where $v_{2}^{T}(t)=-\int \frac{\partial \theta(t)}{\partial t} / \frac{\partial r_{2}^{T}}{\partial v_{2}^{T}} d t$ and $v_{2}^{T}\left(t_{2}^{T}\right)$ is given by Eq. 12 .

\subsection{Monopolistic rail fares}

A monopolistic operator chooses the four earliest arrival times $\left\{t_{1}^{R}, t_{1}^{T}, t_{2}^{R}, t_{2}^{T}\right\}$ and both fares to maximize the sum of profits in all four periods ${ }^{6}$ :

$\max \pi_{m}=\left[N_{1}^{T I}+N_{1}^{T I I}\right] f_{1}^{T}+\left[N_{2}^{T I}+N_{2}^{T I I}\right] f_{2}^{T}$

Note that, since we have expressed the numbers of commuters using each mode as a function of the arrival times of all groups of commuters, using the Wardropian user equilibrium conditions, these four arrival times are now decision variables, instead of the original commuter numbers. Since fares are timeinvariant, no further temporal user equilibrium constraints are necessary, because they have already been substituted in the total commuter numbers. However, two intermodal user equilibrium constraints are needed to ensure that, for users from both nodes, the generalized prices of both modes are equal. These constraints can be evaluated at a number of points in time, but, since all groups commuters are travelling at $t=t^{*}$, it is convenient to use that point:

$f_{1}^{T}+r_{1}^{T}\left(\bar{v}_{1}^{T}\left(t_{1}^{T}, t_{2}^{T}\right)\right)=f_{1}^{R}+r_{1}^{R}\left(\bar{v}_{1}^{R}\left(t_{1}^{R}, t_{2}^{R}\right)\right)$

$f_{2}^{T}+r_{2}^{T}\left(\bar{v}_{2}^{T}\left(t_{1}^{T}, t_{2}^{T}\right)\right)=f_{2}^{R}+r_{2}^{R}\left(\bar{v}_{2}^{R}\left(t_{1}^{R}, t_{2}^{R}\right)\right)$

Where $\bar{v}_{l}^{m}$ is the flow on mode $m$, link $l$ at $t=t^{*}$

The final two constraints ensure that all commuters travel:

$N_{1}^{T I}+N_{1}^{T I I}+N_{1}^{R I}+N_{1}^{R I I}=N$

$N_{2}^{T I}+N_{2}^{T I I}+N_{2}^{R I}+N_{2}^{R I I}=2 N$

The Lagrangian for profit maximalization then becomes:

$$
\begin{aligned}
L_{m}=\pi_{m}+\lambda_{1}[ & \left.f_{1}^{T}+r_{1}^{T}\left(\bar{v}_{1}^{T}\right)-f_{1}^{R}-r_{1}^{R}\left(\bar{v}_{1}^{R}\right)\right]+\lambda_{2}\left[f_{2}^{T}+r_{2}^{T}\left(\bar{v}_{2}^{T}\right)-f_{2}^{R}-r_{2}^{R}\left(\bar{v}_{2}^{R}\right)\right] \\
& +\lambda_{3}\left[N_{1}^{T I}+N_{1}^{T I I}+N_{1}^{R I}+N_{1}^{R I I}-N\right]+\lambda_{4}\left[N_{2}^{T I}+N_{2}^{T I I}+N_{2}^{R I}+N_{2}^{R I I}-2 N\right]
\end{aligned}
$$

Where $\lambda_{1}$ to $\lambda_{4}$ are the Lagrangian multipliers associated with constraints (20)-(23). The relevant firstorder conditions are:

$$
\begin{aligned}
& \frac{\partial L_{m}}{\partial f_{1}^{T}}=N_{1}^{T I}+N_{1}^{T I I}+\lambda_{1}=0 \\
& \frac{\partial L_{m}}{\partial f_{2}^{T}}=N_{2}^{T I}+N_{2}^{T I I}+\lambda_{2}=0 \\
& \frac{\partial L_{m}}{\partial t_{1}^{R}}=-\lambda_{1} \frac{\partial r_{1}^{R}}{\partial \bar{v}_{1}^{R}} \frac{\partial \bar{v}_{1}^{R}}{\partial t_{1}^{R}}-\lambda_{2} \frac{\partial r_{2}^{R}}{\partial \bar{v}_{2}^{R}} \frac{\partial \bar{v}_{2}^{R}}{\partial t_{1}^{R}}+\lambda_{3}\left[\frac{\partial N_{1}^{R I}}{\partial t_{1}^{R}}+\frac{\partial N_{1}^{R I I}}{\partial t_{1}^{R}}\right]+\lambda_{4}\left[\frac{\partial N_{2}^{R I}}{\partial t_{1}^{R}}+\frac{\partial N_{2}^{R I I}}{\partial t_{1}^{R}}\right]=0 \\
& \frac{\partial L_{m}}{\partial t_{1}^{T}}=f_{1}^{T}\left[\frac{\partial N_{1}^{T I}}{\partial t_{1}^{T}}+\frac{\partial N_{1}^{T I I}}{\partial t_{1}^{T}}\right]+f_{2}^{T}\left[\frac{\partial N_{2}^{T I}}{\partial t_{1}^{T}}+\frac{\partial N_{2}^{T I I}}{\partial t_{1}^{T}}\right]+\lambda_{1}\left[\frac{\partial r_{1}^{T}}{\partial \bar{v}_{1}^{T}} \frac{\partial \bar{v}_{1}^{T}}{\partial t_{1}^{T}}\right]+\lambda_{2}\left[\frac{\partial r_{2}^{T}}{\partial \bar{v}_{2}^{T}} \frac{\partial \bar{v}_{2}^{T}}{\partial t_{1}^{T}}\right] \\
& +\lambda_{3}\left[\frac{\partial N_{1}^{T I}}{\partial t_{1}^{T}}+\frac{\partial N_{1}^{T I I}}{\partial t_{1}^{T}}\right]+\lambda_{4}\left[\frac{\partial N_{2}^{T I}}{\partial t_{1}^{T}}+\frac{\partial N_{2}^{T I I}}{\partial t_{1}^{T}}\right]=0
\end{aligned}
$$

\footnotetext{
${ }^{6}$ For simplicity, we assume that costs have no influence on the difference between the optimal monopolistic and duopolistic prices, and thus that there are no economies of scale in the number of links owned. If this were not the case, general conclusions are impossible (see also the static model above).
} 


$$
\begin{gathered}
\frac{\partial L_{m}}{\partial t_{2}^{R}}=-\lambda_{1} \frac{\partial r_{1}^{R}}{\partial \bar{v}_{1}^{R}} \frac{\partial \bar{v}_{1}^{R}}{\partial t_{2}^{R}}-\lambda_{2} \frac{\partial r_{2}^{R}}{\partial \bar{v}_{2}^{R}} \frac{\partial \bar{v}_{2}^{R}}{\partial t_{2}^{R}}+\lambda_{3}\left[\frac{\partial N_{1}^{R I}}{\partial t_{2}^{R}}+\frac{\partial N_{1}^{R I I}}{\partial t_{2}^{R}}\right]+\lambda_{4}\left[\frac{\partial N_{2}^{R I}}{\partial t_{2}^{R}}+\frac{\partial N_{2}^{R I I}}{\partial t_{2}^{R}}\right]=0 \\
\frac{\partial L_{m}}{\partial t_{2}^{T}}=f_{1}^{T}\left[\frac{\partial N_{1}^{T I}}{\partial t_{2}^{T}}+\frac{\partial N_{1}^{T I I}}{\partial t_{2}^{T}}\right]+f_{2}^{T}\left[\frac{\partial N_{2}^{T I}}{\partial t_{2}^{T}}+\frac{\partial N_{2}^{T I I}}{\partial t_{2}^{T}}\right]+\lambda_{1}\left[\frac{\partial r_{1}^{T}}{\partial \bar{v}_{1}^{T}} \frac{\partial \bar{v}_{1}^{T}}{\partial t_{2}^{T}}\right]+\lambda_{2}\left[\frac{\partial r_{2}^{T}}{\partial \bar{v}_{2}^{T}} \frac{\partial \bar{v}_{2}^{T}}{\partial t_{2}^{T}}\right] \\
+\lambda_{3}\left[\frac{\partial N_{1}^{T I}}{\partial t_{2}^{T}}+\frac{\partial N_{1}^{T I I}}{\partial t_{2}^{T}}\right]+\lambda_{4}\left[\frac{\partial N_{2}^{T I}}{\partial t_{2}^{T}}+\frac{\partial N_{2}^{T I}}{\partial t_{2}^{T}}\right]=0
\end{gathered}
$$

Using Eqs. (29)-(34), it is possible to obtain closed-form sultions for $f_{1}^{T}$ and $f_{2}^{T}$, however, the resulting expressions are very tedious, and economic interpretation is therefore hard to give.

\subsection{Duopolistic rail fares}

A Bertrand operator on the first segment maximizes its own profit, subject to the same constraints as the monopolistic operator. The relevant first-order conditions for the two duopolists are very similar to the ones for the monopolist; Eqs. (25)-(27) and (30) are unchanged, although the shadow prices are different. Eqs. (28) and (29) are replaced by:

$$
\begin{aligned}
& \frac{\partial L_{b 1}}{\partial t_{1}^{T}}=f_{1}^{T}\left[\frac{\partial N_{1}^{T I}}{\partial t_{1}^{T}}+\frac{\partial N_{1}^{T I I}}{\partial t_{1}^{T}}\right]+\gamma_{1}\left[\frac{\partial r_{1}^{T}}{\partial \bar{v}_{1}^{T}} \frac{\partial \bar{v}_{1}^{T}}{\partial t_{1}^{T}}\right]+\gamma_{2}\left[\frac{\partial r_{2}^{T}}{\partial \bar{v}_{2}^{T}} \frac{\partial \bar{v}_{2}^{T}}{\partial t_{1}^{T}}\right]+\gamma_{3}\left[\frac{\partial N_{1}^{T I}}{\partial t_{1}^{T}}+\frac{\partial N_{1}^{T I I}}{\partial t_{1}^{T}}\right] \\
& +\gamma_{4}\left[\frac{\partial N_{2}^{T I}}{\partial t_{1}}+\frac{\partial N_{2}^{T I I}}{\partial t_{1}}\right]=0 \\
& \frac{\partial L_{b 1}}{\partial t_{2}^{T}}=f_{1}^{T}\left[\frac{\partial N_{1}^{T I}}{\partial t_{2}^{T}}+\frac{\partial N_{1}^{T I I}}{\partial t_{2}^{T}}\right]+\gamma_{1}\left[\frac{\partial r_{1}^{T}}{\partial \bar{v}_{1}^{T}} \frac{\partial \bar{v}_{1}^{T}}{\partial t_{2}^{T}}\right]+\gamma_{2}\left[\frac{\partial r_{2}^{T}}{\partial \bar{v}_{2}^{T}} \frac{\partial \bar{v}_{2}^{T}}{\partial t_{2}^{T}}\right]+\gamma_{3}\left[\frac{\partial N_{1}^{T I}}{\partial t_{2}^{T}}+\frac{\partial N_{1}^{T I I}}{\partial t_{2}^{T}}\right] \\
& +\gamma_{4}\left[\frac{\partial N_{2}^{T I}}{\partial t_{3}}+\frac{\partial N_{2}^{T I I}}{\partial t_{3}}\right]=0 \\
& \frac{\partial L_{b 2}}{\partial t_{1}^{T}}=f_{2}^{T}\left[\frac{\partial N_{2}^{T I}}{\partial t_{1}^{T}}+\frac{\partial N_{2}^{T I I}}{\partial t_{1}^{T}}\right]+\varphi_{1}\left[\frac{\partial r_{1}^{T}}{\partial \bar{v}_{1}^{T}} \frac{\partial \bar{v}_{1}^{T}}{\partial t_{1}^{T}}\right]+\varphi_{2}\left[\frac{\partial r_{2}^{T}}{\partial \bar{v}_{2}^{T}} \frac{\partial \bar{v}_{2}^{T}}{\partial t_{1}^{T}}\right]+\varphi_{3}\left[\frac{\partial N_{1}^{T I}}{\partial t_{1}^{T}}+\frac{\partial N_{1}^{T I I}}{\partial t_{1}^{T}}\right] \\
& +\varphi_{4}\left[\frac{\partial N_{2}^{T I}}{\partial t_{1}}+\frac{\partial N_{2}^{T I I}}{\partial t_{1}}\right]=0 \\
& \frac{\partial L_{b 2}}{\partial t_{2}^{T}}=f_{2}^{T}\left[\frac{\partial N_{2}^{T I}}{\partial t_{2}^{T}}+\frac{\partial N_{2}^{T I I}}{\partial t_{2}^{T}}\right]+\varphi_{1}\left[\frac{\partial r_{1}^{T}}{\partial \bar{v}_{1}^{T}} \frac{\partial \bar{v}_{1}^{T}}{\partial t_{2}^{T}}\right]+\varphi_{2}\left[\frac{\partial r_{2}^{T}}{\partial \bar{v}_{2}^{T}} \frac{\partial \bar{v}_{2}^{T}}{\partial t_{2}^{T}}\right]+\varphi_{3}\left[\frac{\partial N_{1}^{T I}}{\partial t_{2}^{T}}+\frac{\partial N_{1}^{T I I}}{\partial t_{2}^{T}}\right] \\
& +\varphi_{4}\left[\frac{\partial N_{2}^{T I}}{\partial t_{2}^{T}}+\frac{\partial N_{2}^{T I I}}{\partial t_{2}^{T}}\right]=0
\end{aligned}
$$

Again, it is possible to solve for $f_{1}^{T}$ and $f_{2}^{T}$, but this does not yield helpful results.

\subsection{Fare comparison and comparison with static model}

Although, in this general setting, it is not possible to determine in which cases the fare differentials between the monopolistic and duopolistic settings is positive or negative, the difference between Eqs. (28) - (29) and (32) - (34) clearly shows that there is no reason that this differential will be zero, as it was in the static model. Each duopolist disregards the effect that a change in its fare will have on its competitor's patronage. Fares on one link influence patronage on the other, because a change in one fare affects the trip timing of all commuters using that link, which indirectly affects the trip timing of all commuters, and therefore all generalized prices. A monopolist internalizes these effects.

This phenomenon is not unrelated to the 'double marginalization' in static networks with pricesensitive demand, but it is different. In that case, competitors reduce demand to a level below the social 
optimum, by charging fares that are too high. Here, competitors shift demand to sub-optimal times, by charging fares that, in principle, could be either too high or too low. Naturally then, a static model does not capture this effect, as it disregards trip timing, and hence only allows for inefficiencies in the total commuter flow rather than the flow at any point in time. If demand is fixed, the monopolist's fare setting problem is no longer perfectly separable in two duopolistic problems if there are possibilities for intertemporal substitution. If demand is price-sensitive, both the total number of commuters using each mode and their arrival times can be suboptimal; both the effect discussed above and the static 'double marginalization' are present. From the above, however, it is not obvious whether these effects work in the same direction, or may partly cancel each other.

\subsection{Towards a general network}

The above results, and the reduced-form optimization problem used to obtain them, also apply to a more general multi-modal many-to-one commuter network. The only assumption needed is that, in equilibrium, all routes are used at least some time. As long as this is the case, adding more serial origin nodes, or adding more parallel modes between all nodes will not qualitatively change the results. It is also possible to allow the total number of commuters per node to differ across nodes. Although this will complicate the derivations, the qualitative results will again not change.

It is significantly more difficult to also allow for travel to more destinations; for example, from node 1 to 2 . In this case, travel time growth on a downstream link cannot at the same time compensate for schedule delay cost developments for travellers using only that link, and travellers using that link together with other links, when travel delays are time-varying. This would lead to a temporal separation of travellers from different OD-pairs, and different models have to be developed to address this. It is also difficult to allow for other network structures, where not all nodes can be placed on a straight line; in that case, establishing an arrival order ex-ante may be impossible, and numerical simulations would have to be performed for every possible combination. Also for the present setting, however, numerical modelling can produce additional insights, as we will see in the next section.

\section{Numerical analysis}

The above analysis shows how a reduced-form of the dynamic user equilibrium can be derived analystically. This reduced form was then used to prove that, in a dynamic model, there is likely to be a difference between monopolistic and duopolistic fares. It is possible to obtain closed-form expressions for the user equilibrium flows, as functions of the fares. However, these allow for little, if any, economic interpretation. Closed-form expressions for the optimal fares are more difficult to obtain, and even more difficult to interpret. It is not possible to derive whether this difference is positive or negative, nor how it is influenced by the various model parameters, without specifying the congestion functions $r_{l}^{m}\left(v_{l}^{m}\right)$, and the schedule delay function $\theta(t)$. However, these questions are important, particularly to assess whether they lead to fares and commuter flows that are further away from the optimum, or whether they could cancel out some of the negative effects of the traditional static 'double marginalization'-phenomenon in a more realistic network setting with price-sensitive demand.

We therefore present the results of a numerical analysis, and use those results to identify how the various model parameters influence optimal monopolistic and duopolistic fares. Although much of the previous literature has focused on fares only, it is equally important to examine the welfare implications of the two market structures. To do so, we also consider the relative social costs under both market structures; since demand is fixed, this is the equivalent of social surplus. 


\subsection{Functions and base case parameters}

Train speeds, $S^{T}$, are constant. However, commuters face in-vehicle crowding costs, which we assume to increase linearly in the commuter flow. Fares are constant over time. A traveller's total costs of travelling on a link are then:

$r_{l}^{T}(t)+f_{l}^{T}=\alpha \frac{m}{S^{T}}+m \xi_{1} v_{l}^{T}(t)+f_{l}^{T}$

Where $\alpha$ is the value of travel time, $m$ the segment length, and $\xi_{1}$ a parameter. Link-based congestion costs for road users consist only of the costs of travel time and, as before, roads are not priced:

$r_{l}^{R}(t)=\alpha \frac{m}{s_{l}^{R}(t)}$

where $s_{l}^{R}(t)$ is the road speed on segment $l$ at time $t$. Speeds increase linearly in the commuter flow ${ }^{7}$, with a maximum (free-flow) speed $S^{R}$ :

$\frac{1}{s_{l}^{R}(t)}=\frac{1}{S^{R}}+\xi_{2} v_{l}^{R}(t)$

Where $\xi_{2}$ is a parameter.

Finally, schedule delay costs $\theta(t)$ are linear in $t$ :

$\theta(t)=\beta\left[t^{*}-t\right]$

where $\beta$ is the value of schedule delay early. As before, late arrivals are prohibited. Base case parameters are listed in Table 3; they are chosen to result in realistic fares and costs of congestion.

Table 3 - Base case parameters

\begin{tabular}{cc}
\hline \hline Parameter & Value \\
\hline$\alpha$ & $5 € / \mathrm{hr}$ \\
$\beta$ & $3 € / \mathrm{hr}$ \\
$m$ & $10 \mathrm{~km}$ \\
$S^{T}$ & $100 \mathrm{~km} / \mathrm{h}$ \\
$S^{R}$ & $120 \mathrm{~km} / \mathrm{h}$ \\
$\xi_{1}$ & $0.002[€ / \mathrm{pass}.][\mathrm{km} / \mathrm{h}]^{-1}$ \\
$\xi_{2}$ & $0.002[\text { pass. } / \mathrm{hr}]^{-1}[\mathrm{~km} / \mathrm{h}]^{-1}$ \\
$N$ & 100 pass. \\
\hline \hline
\end{tabular}

For simplicity, we assume that the marginal costs of transporting passengers is zero; assuming a constant positive marginal cost would complicate the analysis without qualitatively changing the results.

\subsection{Value of time and value of schedule delay}

We first vary the value of schedule delay, fixing all other parameters to the values listed in Table 2; in effect, we therefore vary the ratio between the value of schedule delay and the value of time. The left panel in Fig. 2 shows the difference between the duopolistic and monopolistic price for each segment, as a fraction of the monopolistic price. The right panel shows the social cost difference as a fraction of monopolistic welfare; positive values indicate that social costs are higher in a duopoly.

\footnotetext{
${ }^{7}$ I.e., there is flow congestion as in Chu (1995). Since the network consists of two serial segments, the more popular bottleneck congestion is difficult to implement. For simplicity, the congestion function is linear; there is no hypercongestion.
} 

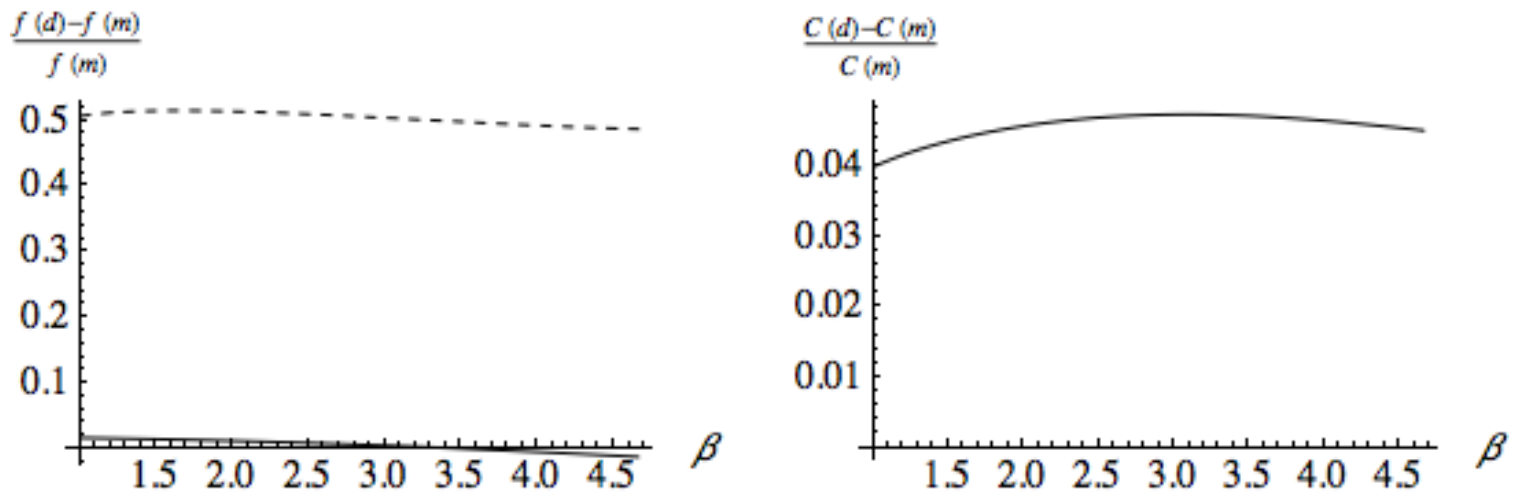

------- Segment $1 \longrightarrow$ Segment 2

Figure 2 - Varying the value of schedule delay

An increase in $\beta$ has two effects. Since being early at work is now more expensive, commuters will want to arrive closer to their desired arrival time, and the total network-wide congestion costs will be higher (see Eqs. (9) - (15) above, where now $\partial \theta(t) / \partial t=\beta$ ). Both the monopolist and the duopolists take advantage of the increased level of congestion to raise fares, but the duopolists disregard the effect on each other's patronage, and consequently raise fares by a higher amount than the monopolist. This increases the fare differential. However, an increased value of schedule affects rail and road users in different ways. While, in this linear model, road travel flows increase at rate $\beta / 2 \alpha m \xi_{2}$ in period RI and twice as fast in period RII (see Eqs. (9) and (37)-(38)), rail flows increase at rate $\beta / 2 m \xi_{1}$ in period TI and twice as fast in period TII (see Eqs. (12) and (36)). Since rail flows increase at a higher rate to begin with, an increase in the value of schedule delay also has a higher impact on train users. Hence, an increase in $\beta$ reduces the market power of rail operators, and thus the tendency of the duopolists to charge higher fares, which reduces the price differential. As Fig. 2 shows, the second effect is clearly stronger on the more heavily congested downstream segment, as well as on the downstream segment for large values of $\beta$. The first effect dominates for very small values of $\beta$. However, both are small; although the relative fare differences do vary in $\beta$, they do not vary by large amounts.

The figure also shows that, whatever the value of schedule delay, the fare differential is much higher on the upstream segment. This results from the fact that all fares are lower on this segment due to the lower number of commuter traversing it; the same absolute fare difference therefore leads to much higher fractions on the upstream segment.

Finally, although the difference is small in relative terms, the duopolistic fare on the downstream segment can be lower than the monopolistic fare. This is more likely to happen as the value of schedule delay approaches the value of time, and implies that commuters from node 2 can be worse off in the monopolistic situation. This is an important observation, as it is a clear difference with the static 'double marginalization'-effect; while the latter can only lead to higher duopolistic fares, the dynamic effect can apparently reduce fares and hence, work in the opposite direction. However, although the numerical results therefore confirm the analytical finding that the duopolistic fare may be smaller than the monopolistic fare, this effect may have a limited quantitative impact in practice, despite its qualitative significance. The right panel in Fig. 2 also shows that, for society as a whole, a monopoly is still 
preferable. Although the relative social costs do vary with the value of schedule delay, as a result of varying fares and commuter flows, social costs remain between $4 \%$ and $5 \%$ higher in the duopolistic situation.

\subsection{Congestion}

Fig. 3 shows the results of a similar exercise, in which $\xi_{1}$, the rail congestion parameter, is varied from $1 / 1000$ to $1 / 100$. This parameter multiplies the commuter flow in the link-based rail cost functions, so an increase in $\xi_{1}$ increases the cost of in-vehicle crowding. Since $\xi_{2}$ is held constant, this increases the importance of congestion in the train relative to the importance of congestion on the road. We can therefore again expect two effects: firstly, as $\xi_{1}$ increases, rail operators face tougher competition from the road, which will decrease fares, and the tendency of duopolistic competitors to charge higher fares than a monopolist. However, the congestion costs increase in the whole network, as commuters equate the generalized prices of the two nodes. This increases the tendency of duopolistic competitors to charge higher fares.
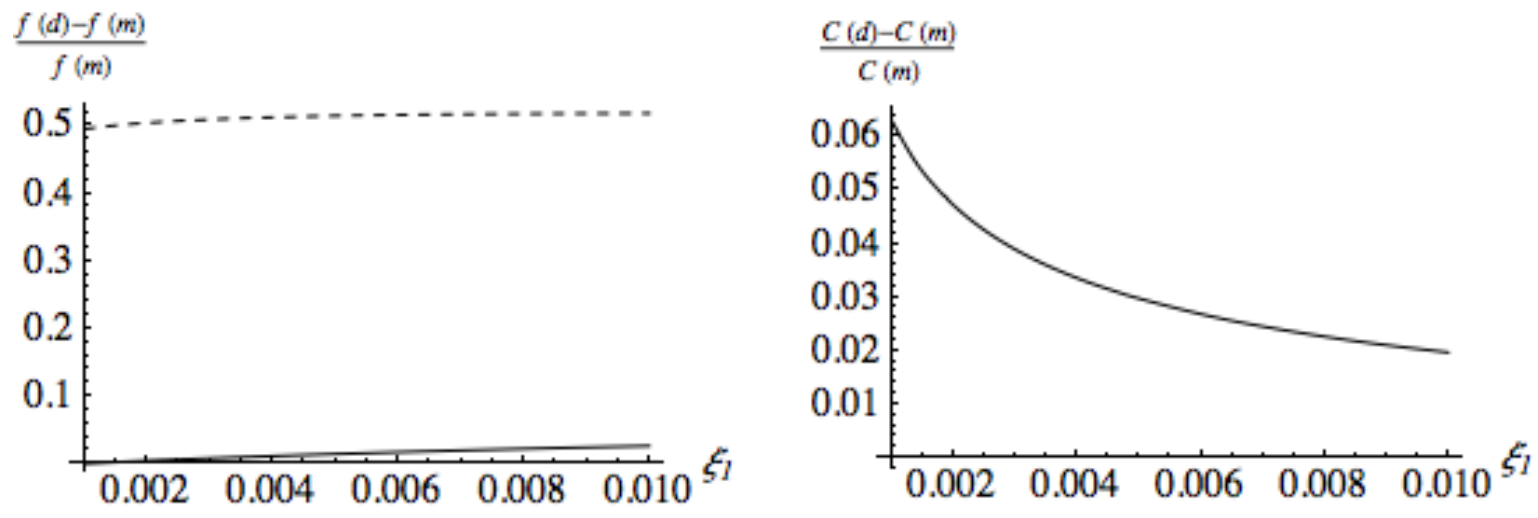

------ Segment $1 \longrightarrow$ Segment 2

Figure 3 - Varying the costs of congestion

All fares do indeed decrease in $\xi_{1}$; as congestion in the train is more costly, competition from the road increases, and rail fares subsequently decrease. The relative fare difference between duopoly and monopoly, however, increases in $\xi_{1}$ on both segments; the total congestion effect is, in this case, stronger. Again, however, this effect is relatively small in the observed range. Again, also, a monopoly is always more efficient than a duopoly, although the cost difference between the two is very small for a very high unit cost of congestion. This shows that the social surplus effects of a parameter change can be rather different from the effects on fares.

\subsection{Congestion and value of schedule delay}

So far, we have considered the influence of the congestion parameter and the value of schedule delay separately. Of course, there are also interaction effects between these two parameters. Fig. 4 therefore shows the effects of a change in the value of schedule delay for a congestion parameter twice as high as the value used in Fig. $2\left(\xi_{1}=0.004\right)$. 

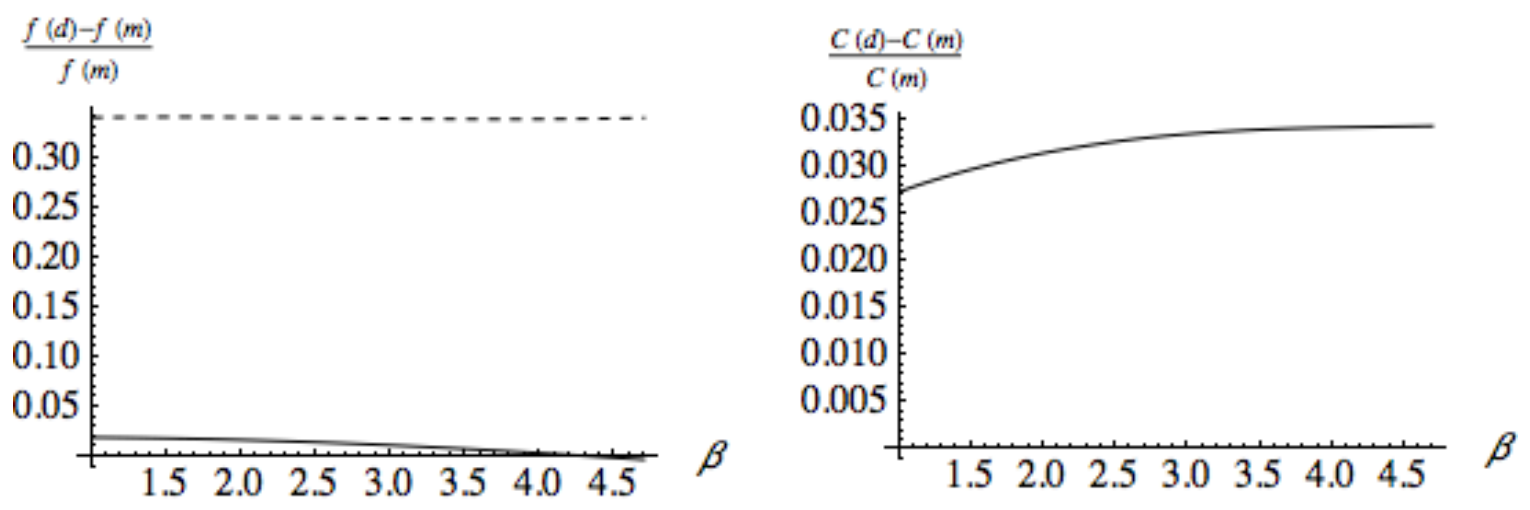

Figure 4 - Varying the value of schedule delay $\left(\xi_{1}=0.004\right)$

Comparing Figs. 2 and 4, it is clear that this interaction effect is important. With this higher congestion parameter, the first, direct effect of an increase in $\beta$ on congestion (see above) and thus on the ability of operators to charge higher fares, is much more important. However, the ranges of relative fare differences have not changed much. The influence of the value of schedule delay on the fare differential is still limited and social cost differences, although lower, also do not change by large amounts when $\beta$ is varied.

\subsection{Commuter numbers}

Fig. 5 shows the results of a simulation in which the number of commuters departing from each node was increased to up to ten times the base case value. Naturally, all fares increase in the number of commuters, as an increased number of commuters increases the congestion costs in the system. Both the monopolist and the duopolists are able to increase their fares as a result of a higher number of commuters. The duopolists, disregarding the externality they impose on their competitor's patronage, increase their fares more than the monopolist; hence, absolute fare differences increase in $N$. However, the relative fare difference changes very little, and the same is true for the relative welfare difference. 

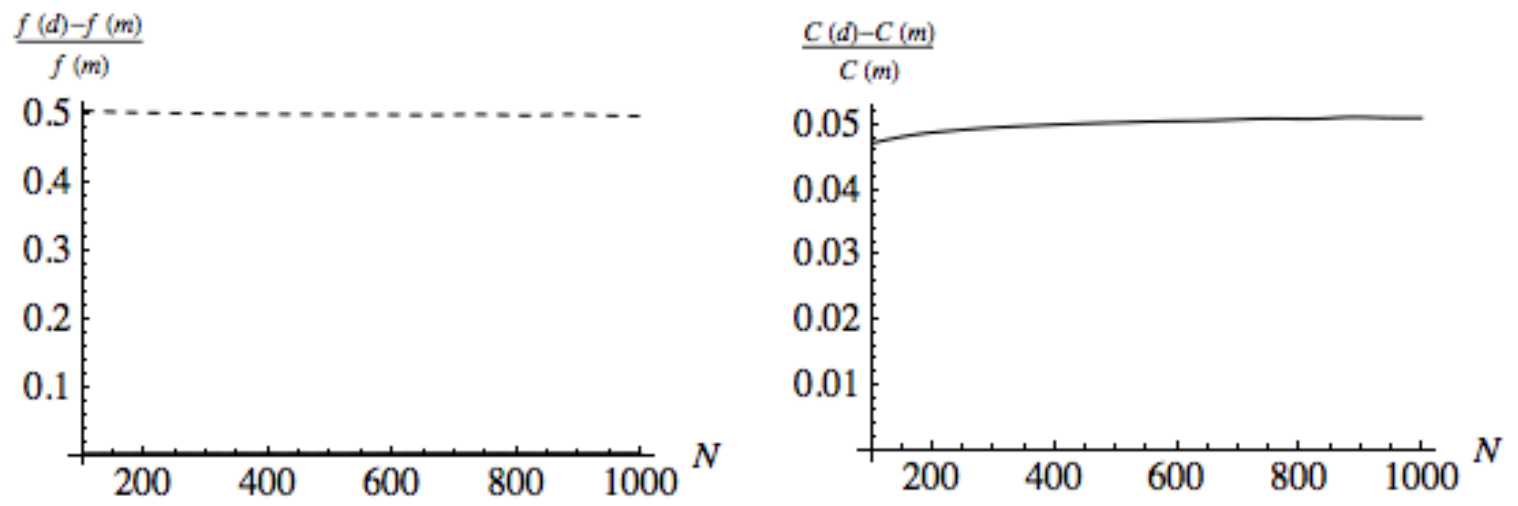

Segment 1

Segment 2

Figure 5 - Varying the number of commuters

\subsection{Numerical conclusions}

The relative fares and relative cost differences appear to be remarkably robust in this numerical exercise. That suggests that lessons on desirable market organization one can draw for this particular spatial setup are quite generic, which is good news is policies are to be developed in a changing world or under conditions of uncertainty on parameter values.

\section{Conclusions}

We have shown that, in a multi-modal many-to-one commuter network where rail operators compete with unpriced roads, serial competition can influence fares, even in the absence of elastic demand. This results from the fact that, in a dynamic model, commuters have possibilities for intertemporal substitution, even if they do not have the option not to travel. Therefore, a fare change on one rail link changes not only the number of rail commuters relative to the number of road commuters, but also their trip timing decisions. If demand is fixed, the former will not necessarily affect anyone travelling on other links in the network, as we have shown with a simple static model, but the latter will change passengers flows in the whole network and through those, other operators' patronages and profits. Naturally, a monopolistic operator internalizes the effect a price increase on one link has on the patronage of the other links, but a Bertrand-Nash operator disregards this, which leads to different, and potentially higher fares.

However, compared to static models, dynamic models are much more difficult to solve. For the many-to-one network described above, we have derived a reduced form under the assumption that fares are time-invariant, in which the rail operators optimize their fares and the boundaries of arrival time intervals, rather than the commuter flows. This makes subsequent calculations considerably easier, although general analytical solutions are still difficult to obtain.

Our numerical simulations show that, although duopolistic fares are higher than monopolistic for a wide range of parameters, this need not always be the case. Especially if the value of time approaches the value of schedule delay, and the unit cost of congestion in the train is higher than on the road, fares on at least one link may in fact be lower in a duopoly. This is a distinct difference with the static 'double marginalization'-effect observed in models with price-sensitive demand. However, the simulations also 
show that model parameters, such as the value of schedule delay and the cost of congestion have only a limited effect on the fare difference between a monopoly and a duopoly and on the differences in social costs. This indicates that the conclusions drawn above are robust to assumption changes, and may also apply in many other spatial setups.

More research is necessary to explore these issues in at least three directions. Firstly, the effect of ownership on the substitute mode (the road) should be examined, especially in situations where the road is owned by a public operator, who sets a second-best road price to offset the negative effects of serial competition in the rail market. Secondly, as the numerical simulation shows that the fare increases may not affect all groups of commuters equally, local policy makers may try to influence the rail market to compensate the commuters in their area by, for example, subsidising rail commuters from travelling from one node, or limiting the number of train services on one link. This could be modelled by adding an additional level, in which local governments maximize the social benefit in one node only by manipulating the variables over which they have control. Finally, a more general network setting, in which there is more than one destination, should be explored, as spatial interactions are clearly important.

However, despite the relative simplicity of the models outlined above, they do show that it is important to consider the effects of market structures in a situation where rail operators compete with an unpriced road, and to consider them in a dynamic setting. This introduces another reason for fare differences between different forms of competition, through the commuter's departure timing choices. As we have shown, although these effects often work in the same direction as the classic 'double marginalization' that occurs when demand is elastic, there is a potential for it to work in the opposite direction. In any case, it should not be ignored, and we have shown ways to facilitate implementation.

\section{Acknowledgements}

Financial support from ERC Advanced Grant \#246969 is gratefully acknowledged. The authors thank Hugo Silva, Sergej Gubins, and the participants of the 2011 Kuhmo Nectar conference, for helpful comments on an earlier version of this paper.

\section{References}

Arnott, R., DePalma, E., 2011. The corridor problem: preliminary results on the no-toll equilibrium. Transportation Research Part B 45(5), 743-768.

Arnott, R., Yan, A., 2000. The two-mode problem: second-best pricing and capacity. Review of Urban \& Regional Development Studies 12(3), 170-199.

Chu, X., 1995. Endogenous trip scheduling: the Henderson approach reformulated and compared with the Vickrey approach. Journal of Urban Economics 37(3), 324-343.

De Borger, B., Dunkerley, F., Proost, S., 2008. The interaction between tolls and capacity investment in serial and parallel transport networks. Review of Network Economics 7(1), 136-158.

Economides, N., Salop, S.C., 1992. Competition and integration among complements, and network market structure. Journal of Industrial Economics 40(1), 105-123.

Henderson, J.V., 1974. Road congestion: a reconsideration of pricing theory. Journal of Urban Economics 1(3), 346-365.

Pels, E., Verhoef, E.T., 2007. Infrastructure pricing and competition between modes in urban transport. Environment and Planning A 39(9), 2119-2138.

Small, K., 1982. The scheduling of consumer activities: work trips. American Economic Review 72(3), 467-479. 
Tian, Q., Huang, H.-J., Yang, H, 2007. Equilibrium properties of the morning peak-period commuting in a many-to-one mass transit system. Transportation Research Part B 41(6), 616-631.

Verhoef, E.T., 2008. Private roads: auctions and competition in networks. Journal of Transport Economics and Policy 42(3), 463-493.

Wardrop, J.G., 1952. Some theoretical aspects of road traffic research. Proceedings, Institute of Civil Engineers Part II 1, 325-378. 